\title{
La susceptibilidad magnética en materiales superconductores
}

\author{
J. C. González ${ }^{* a}$, A. Osorio ${ }^{b}$, A. Bustamante ${ }^{\mathrm{a}}$ \\ ${ }^{a}$ Laboratorio de Superconductividad, Facultad de Ciencias Físicas - UNMSM. Ciudad Universitaria, Lima 1, Perú. \\ ${ }^{b}$ Facultad de Química e Ing. Química - UNMSM. Ciudad Universitaria, Lima 1, Perú.
}

Recibido:25-09-2009; Aceptado: 19-11-2009

\begin{abstract}
Resumen
Llevados por la demanda de comprender y cuantificar las propiedades magnéticas de materiales superconductores, la susceptibilidad magnética $\mathrm{AC}$ y DC ha emergido como una técnica de gran valor en el campo de la superconductividad; permitiéndonos cuantificar: la temperatura crítica superconductora, las fracciones: Meissner y blindaje diamagnético, la constante de Curie, la temperatura de Curie-Weiss y el magnetón de Bohr efectivo.
\end{abstract}

Palabras clave: Susceptibilidad magnética, Fracción Meissner, Blindaje diamagnético, Constante de Curie, Temperatura de Curie-Weiss.

\section{Magnetic susceptibility in the superconducting materials}

\begin{abstract}
Driven by the demand to understand and quantify the magnetic properties of superconducting materials, the acand dc-magnetic susceptibility has emerged as a valuable technique in the superconductivity field; allowed us to quantify: superconducting critical temperature, Meissner and diamagnetic shielding fractions, Curie constant, Curie-Weiss temperature and effective Bohr magnetón.
\end{abstract}

Keywords: Magnetic susceptibility, Meissner fraction, Diamagnetic shielding, Curie constant, Curie-Weiss temperature.

\section{Introducción}

Desde sus inicios la superconductividad se encuentra en la frontera de la innovación en el área de la ciencia y la tecnología [1]. El fenómeno de la superconductividad aparece en ciertos materiales por debajo de una temperatura denominada temperatura crítica superconductora (Tc), caracterizada por una ausencia de resistencia eléctrica $(\rho=0)$ y la exclusión de un débil campo magnético del interior $(\mathbf{B i n t}=0)$.

Se distinguen dos tipos de materiales superconductores. Los de baja y alta Tc. Los superconductores de baja Tc se pueden explicar a partir de la formación de parejas de los portadores de cargas vía la interacción de un fonón, estos materiales son descritos por la teoría desarrollada por Bardeen, Cooper y Schrieffer (teoría BCS) en el año 1957. En cambio los superconductores de alta Tc no pueden ser descritos por el acoplamiento vía electrón-fonón, ni a través de la teoría BCS, teoría que no puede explicar las altas energías de acoplamiento necesarias en estos tipos de superconductores ( $\sim 100 \mathrm{~K})$. Desde el año 1986 fueron descubiertos nuevos materiales superconductores con temperaturas críticas superconductora por encima de los $90 \mathrm{~K}$, los cuales están basados en complicadas secuencias de capas de óxidos [2].

El estudio de las propiedades magnéticas en los materiales superconductores ha generado un

Contacto : abustamanted@unmsm.edu.pe 
creciente interés en el uso de instrumentos para medir la respuesta magnética [3] del material a un campo externo de intensidad magnética. El más popular debido a su sensibilidad, fácil uso y calibración, así como exactitud, es el magnetómetro que contiene el dispositivo SQUID (Superconducting Quantum Interference Device), dicho magnetómetro mide el flujo magnético del momento magnético de la muestra a través de una bobina sensora.

Se presenta los resultados del estudio de la susceptibilidad magnética en superconductores de baja y alta Tc a través de medidas de susceptibilidad magnética molar DC (estática) y $\mathrm{AC}$ (oscilante). El rango de temperatura utilizado entre 5 a $300 \mathrm{~K}$ se dividió en dos: a) 5 $100 \mathrm{~K}$ y b) $100-300 \mathrm{~K}$, permitiéndonos analizar la respuesta magnética en los estados: superconductor y normal respectivamente. Las medidas de susceptibilidad magnética se realizaron en procesos de enfriamiento denominados: enfriamiento con campo (fieldcooled, FC) y sin campo (zero-field-cooled, ZFC).

\section{Fundamento teórico}

\subsection{Susceptibilidad Magnética}

La susceptibilidad magnética de una sustancia homogénea, es el grado de magnetización de un material, en respuesta a un campo magnético externo aplicado. Entonces, se define la susceptibilidad magnética por unidad de volumen, $\chi$, descrita por la siguiente ecuación:

$$
\chi=\frac{\mathbf{M}}{\mathbf{H}_{\mathrm{ext}}}
$$

donde $\mathbf{M}$ es la magnetización del material (la intensidad del momento magnético por unidad de volumen) y $\mathbf{H}_{\text {ext }}$ es la intensidad de campo magnético externo aplicado. Además, la susceptibilidad magnética molar por unidad de volumen se expresa por:

$\chi=\frac{M M[\mathrm{emu}]^{*} \text { Peso Molecular }[\mathrm{g} / \mathrm{mol}]}{\mathrm{H}_{\text {ext }}[\mathrm{Oe}]^{*} \text { Masa }[\mathrm{g}]}$

donde $\mathrm{MM}$ es el momento magnético, $\mathrm{H}_{\mathrm{ext}}$ es el módulo del campo externo aplicado.

Podemos decir que, en general, la intensidad de campo magnético externo aplicado consiste de una componente estática $\mathbf{H}_{0}$ y una componente oscilante $\mathbf{H}_{1}$ y esta expresado en el tiempo, $t$, por:

$$
\mathbf{H}_{\text {ext }}(t)=\mathbf{H}_{0}+\mathbf{H}_{1} \cos \omega t
$$

Entonces, la magnetización de una sustancia en un campo oscilante esta expresada por:

$$
\mathbf{M}(t)=\mathbf{M}_{0}+\mathbf{M}_{1} \cos (\omega t-\varphi)
$$

donde $\varphi$ es la diferencia de fase debido al retraso que sufre la magnetización que ha quedado detrás de la componente oscilante del campo magnético. En términos de las susceptibilidades magnéticas expresadas por la ecuación (1), podemos escribir la magnetización como:

$$
\begin{aligned}
\mathbf{M}(t)=\chi_{0} \mathbf{H}_{0}+\chi^{\prime} \mathbf{H}_{1} & \cos (\omega t)+ \\
& +\chi^{\prime \prime} \mathbf{H}_{1} \operatorname{sen}(\omega t)
\end{aligned}
$$

donde $\chi^{\prime}$ está en fase con el campo aplicado $\mathrm{AC}$, mientras que $\chi^{\prime \prime}$ es la componente fuera de fase. En el estado superconductor $(\mathrm{T}<\mathrm{Tc}) \quad \chi^{\prime}$ refleja la capacidad del apantallamiento del campo magnético (blindaje diamagnético), mientras que $\chi^{\prime \prime}$ es una medida de la irreversibilidad magnética. En particular, para bajas frecuencias la magnetización $\mathbf{M}_{1}$ (ecuación 4) no se retrasa con el campo aplicado, por lo cual $\varphi$ es cero, y en el límite de campos pequeños $\chi^{\prime}=\chi_{0}$, mientras que $\chi^{\prime \prime}=0$.

Regímenes de Enfriamiento: Con y Sin Campo $\underline{\text { Magnético Aplicado }}$

El régimen de enfriamiento con campo aplicado (field-cooled, FC) consiste primero en aplicar a la muestra una intensidad de campo magnético externo, $\mathbf{H}_{\text {ext }}$, débil (típicamente de algunos Oersteds: 2 a 20 Oe) mientras se encuentra en su estado normal, posteriormente se la enfría por debajo de su Tc en presencia del campo aplicado, midiendo la susceptibilidad magnética DC o AC a diferentes temperaturas (figura 1). Bajo esta condición el superconductor exhibe lo que es referido como el efecto Meissner o expulsión del flujo magnético.

Por otro lado, en el régimen de enfriamiento sin campo aplicado (zero-field-cooled, ZFC) consiste primero en enfriar la muestra por debajo de su Tc en ausencia del campo aplicado $\left(\mathbf{H}_{\mathrm{ext}}=0\right)$, posteriormente se aplica una débil intensidad de campo magnético externo $\mathbf{H}_{\text {ext }}$, finalmente se mide la susceptibilidad magnética 
DC o AC a diferentes temperaturas mientras se calienta la muestra (figura 1). Bajo este régimen de enfriamiento, el material superconductor exhibe lo que es llamado el blindaje diamagnético o exclusión de flujo, que significa que el flujo de la intensidad de campo aplicado $\mathbf{H}_{\text {ext }}$ no penetra en la muestra. La susceptibilidad magnética medida tiene un valor negativo, idealmente $-\frac{1}{4 \pi}$ en magnitud (sistema CGS) para una completa exclusión del flujo.

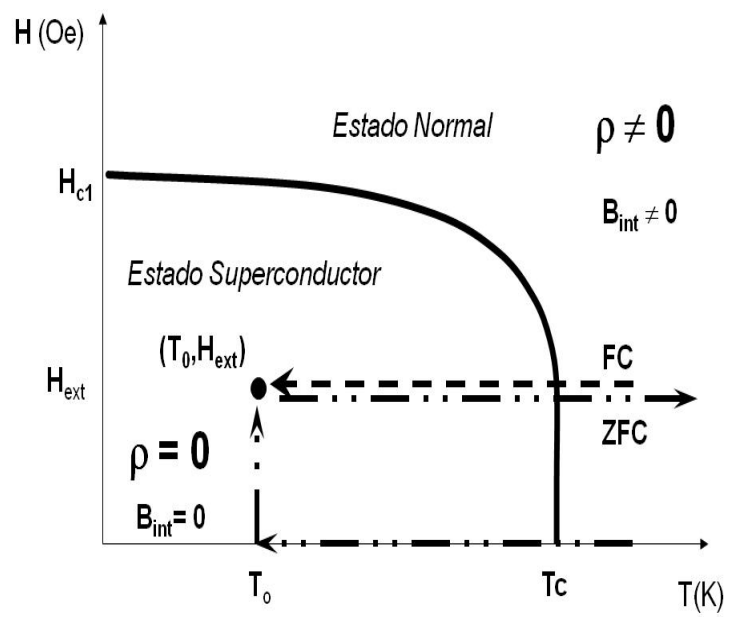

Fig. 1. Regímenes de enfriamiento con campo magnético externo aplicado (FC) y sin campo magnético externo aplicado (ZFC).

\section{$\underline{\text { Fracción Meissner }}$}

El efecto Meissner es la expulsión del flujo de la intensidad de campo magnético externo aplicado al enfriar un superconductor por debajo de su Tc. La expulsión del flujo magnético es el menor estado de energía para el superconductor en un campo magnético externo débil cuyo efecto es la anulación de la inducción magnética interna $\left(\mathbf{B}_{\text {int }}=0\right)$. Es posible calcular la fracción porcentual del volumen superconductor en la muestra o fracción Meissner a través de los datos provenientes de la susceptibilidad magnética, utilizando las medidas del régimen FC, pues una medida de la susceptibilidad magnética en un régimen ZFC descarta cualquier conclusión en el estado Meissner de la muestra, además debido a la naturaleza del régimen $\mathrm{ZFC}$ es posible calcular la fracción de blindaje diamagnético.

\section{Parte experimental}

Para el estudio de la susceptibilidad magnética se utilizaron ocho muestras: (a) un material de baja Tc, Plomo, (b) siete compuestos de alta Tc compuestos por: seis de ellos en forma de polvo $\left[\mathrm{Y}_{0.75}, \mathrm{Ca}_{0.25}\right](\mathrm{Ba}, \mathrm{Sr}) \mathrm{Cu}_{2.80}\left(\mathrm{PO}_{4}\right)_{0.20} \mathrm{O}_{\mathrm{z}} \quad \mathrm{e}$ $\left[\mathrm{Y}_{0.75}, \mathrm{Ca}_{0.25}\right](\mathrm{Ba}, \mathrm{Sr}) \mathrm{Cu}_{3-\mathrm{x}}\left(\mathrm{PO}_{4}\right)_{\mathrm{x}} \mathrm{O}_{\mathrm{z}}$ con $\mathrm{x}=0.0$, $0.15,0.25$ y 0.30 , e $\mathrm{YBa}_{2} \mathrm{Cu}_{3} \mathrm{O}_{7}$, además de una película delgada de $\mathrm{YBa}_{2} \mathrm{Cu}_{3} \mathrm{O}_{7}$. La muestra de $\mathrm{Pb}$ fue obtenida de la firma Alfa Aesar con 99.9\% de pureza, en forma de barra con sección transversal cuadrada $\left(1.0 \mathrm{~mm}^{2}\right)$, longitud 6.5 $\mathrm{mm}$, y una masa de $0.050 \mathrm{~g}$ Las seis muestras en polvo conteniendo fosfatos fueron preparadas mediante la técnica de reacción de estado sólido [4], excepto el $\mathrm{YBa}_{2} \mathrm{Cu}_{3} \mathrm{O}_{7}$ obtenido por el método denominado Sol-Gel [5]. Finalmente la muestra en forma de película delgada de textura biaxial de $\mathrm{YBa}_{2} \mathrm{Cu}_{3} \mathrm{O}_{7}$ crecida sobre monocristal de $\mathrm{LaAlO}_{3}$ con un espesor de $0.250 \mu \mathrm{m}$ se produjo mediante la técnica TFA-MOD [6]. Las medidas de la susceptibilidad magnética DC fueron realizadas en un magnetómetro del tipo MPMS (Magnetic Property Measurement System) modelo XL5 con dispositivo SQUID de la compañía Quantum Design, en el rango de temperaturas de $5-300 \mathrm{~K}$. Mientras que las medidas de magnetización $\mathrm{AC}$ fueron obtenidas mediante un magnetómetro del tipo PPMS (Physical Property Measurement System) con dispositivo SQUID de la Quantum Design en los mismos rangos de trabajo. Los campos externos aplicados varían según la muestra bajo estudio.

\section{Resultados y discusión}

Para comprensión de los resultados obtenidos, esta parte está divida en dos: 1) estado superconductor, y 2) estado normal.

\subsection{Estado Superconductor $(\mathrm{T}<\mathrm{Tc})$.}

a) Muestra Metálica: Los materiales superconductores de baja Tc poseen una región Meissner bien definida por la curva de magnetización (figura 1), que permitió cuantificar la fracción de blindaje diamagnético. En su estado de más baja energía la inducción magnética se anula en el interior de la barra. Las supercorrientes de apantallamiento fluyen en una región cercana a la superficie de la barra para cancelar exactamente el campo aplicado en el interior.

La figura 2 ilustra la magnetización DC de una barra de plomo luego de un proceso ZFC, manteniendo la temperatura externa constante e igual a $5 \mathrm{~K}$ en el rango de 5 a $500 \mathrm{Oe}$, con el campo aplicado paralelo al eje de la barra. 


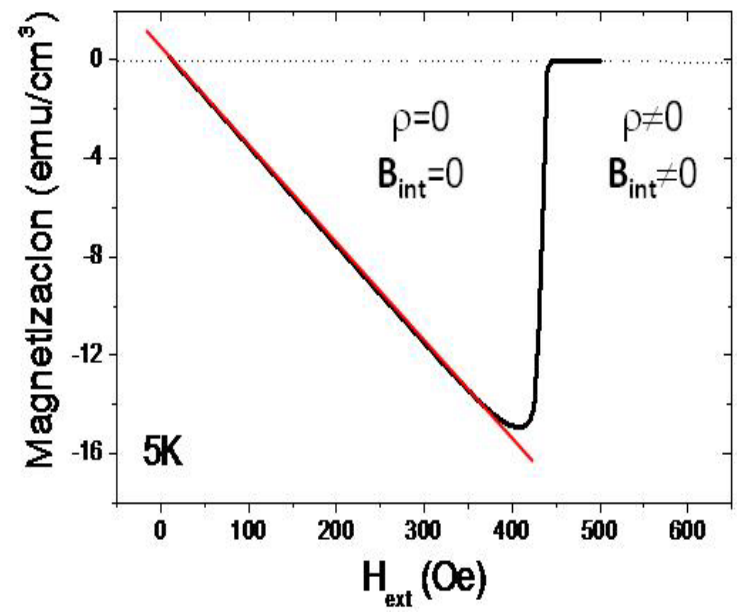

Fig. 2. Magnetización $\mathrm{DC}$ de una barra de $\mathrm{Pb}$ de sección transversal cuadrada a diferentes campos externos aplicados. Ajuste lineal entre 5 a 300 Oe $\left(-4 \pi \chi^{\prime}=0.0397\right)$. En el estado superconductor, la magnitud del flujo de campo magnético, $\mathbf{B}_{\text {int }}$, y la resistividad, $\rho$, se anulan.

Los datos de la figura 2 también nos muestran la magnitud del campo crítico, Hc, intensidad de campo magnético externo aplicado por encima del cual se destruye el fenómeno superconductor. El efecto de blindaje diamagnético en la muestra aparece en respuesta al campo magnético aplicado y la magnetización permanece lineal con la intensidad de campo magnético aplicado hasta que las líneas de flujo magnético empiecen a ingresar dentro de la barra de $\mathrm{Pb}$. Además, el campo crítico $\mathbf{H c}$ está determinado por el punto de inflexión de la curva $\mathrm{M}-\mathrm{H}$, esto sucede a 425 Oe a $5 \mathrm{~K}$. Obsérvese la frontera entre el estado superconductor y el normal, no es aguda sino que posee un ancho finito: $\Delta \mathbf{H}=30 \mathrm{Oe}$.

Cuantificamos la fracción del blindaje diamagnético a la temperatura de $5 \mathrm{~K}$ mediante la razón entre la susceptibilidad magnética experimental $\chi_{\text {Exp }}^{\prime}=-0.0032$ y la magnitud de susceptibilidad teórica $\chi^{\prime}$ Teo $=-0.0822$ (despreciando la profundidad de penetración finita e incluyendo una corrección debido al factor de desmagnetización), donde se ha utilizado el factor de desmagnetización para la muestra de $\mathrm{Pb}$ debido a su geometría [7] es igual a 0.392. Por lo tanto, la fracción de blindaje diamagnético es igual al $4 \%$, descrito por la siguiente relación:

$$
\frac{\chi_{\text {Exp }}^{\prime}}{\chi_{\text {Teo }}^{\prime}} \times 100=4 \%
$$

b) Muestras en Polvo conteniendo Fosfatos: La figura 3 muestra las medidas de susceptibilidad DC de $\left[\mathrm{Y}_{0.75}, \mathrm{Ca}_{0.25}\right](\mathrm{Ba}, \mathrm{Sr}) \mathrm{Cu}_{2.75}\left(\mathrm{PO}_{4}\right)_{0.25} \mathrm{O}_{\mathrm{z}}$ en polvo con una intensidad de campo magnético externo aplicado igual a 2 Oe en los dos procesos de enfriamiento: ZFC y FC. El peso molecular del compuesto es $596.92 \mathrm{~g} / \mathrm{mol} \mathrm{y} \mathrm{la}$ masa utilizada en el estudio fue $0.0131 \mathrm{~g}$.

El interés en estudiar esta muestra en procesos de enfriamiento FC y ZFC, fue dirigida para calcular la fracción Meissner y blindaje diamagnético, así como entender la diferencia entre las señales diamagnéticas FC y ZFC.

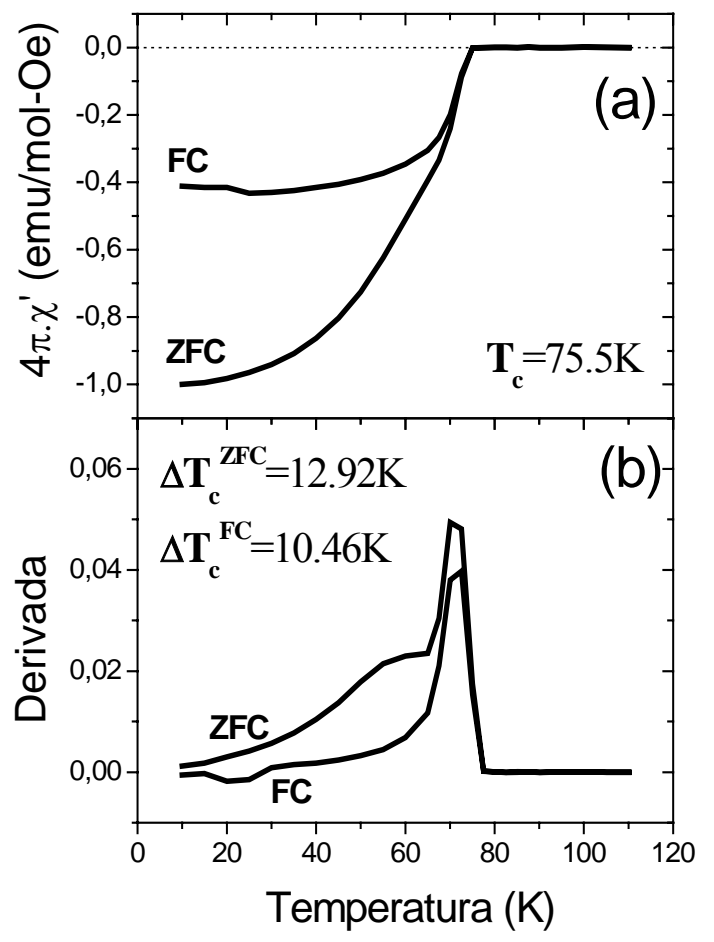

Fig. 3. Susceptibilidad magnética DC para la muestra cerámica superconductora en polvo $\left[\mathrm{Y}_{0.75}, \mathrm{Ca}_{0.25}\right](\mathrm{Ba}, \mathrm{Sr}) \mathrm{Cu}_{2.75}\left(\mathrm{PO}_{4}\right)_{0.25} \mathrm{O}_{\mathrm{z}}$ con una intensidad de campo magnético externo aplicado de 2Oe. (a) susceptibilidad magnética ZFC y FC, (b) primera derivada de las curvas de susceptibilidad en (a).

La primera información que obtenemos es la temperatura crítica superconductora igual a $75.5 \mathrm{~K}$ (figura 3a) mediante una clara señal diamagnética, para ambos procesos de enfriamiento. Utilizamos el criterio de la primera derivada de la señal de susceptibilidad magnética [8] para calcular el ancho finito de la transición al estado superconductor de los dos procesos de enfriamiento a través de la anchura a media altura del pico de la figura $3 \mathrm{~b}$, cuyas magnitudes son: $12.92 \mathrm{~K}$ y $10.46 \mathrm{~K}$, para los procesos ZFC y $\mathrm{FC}$, respectivamente. 
Además se observó una saturación de las señales diamagnética ZFC y FC solamente a bajas temperaturas, indicando la naturaleza granular de la fase superconductora y una superconductividad característica de bulk. Consideramos que las fronteras de grano superconductores forman uniones débiles del tipo Josephson donde la densidad de corriente crítica intergranos e intragranos fluye a través y entre las uniones de Josephson respectivamente, es decir, que las corrientes superconductoras no solamente circulan dentro los granos, sino que también circulan entre los granos, produciendo un efecto de exclusión o expulsión completa del flujo del campo magnético externo a bajas temperaturas $(10 \mathrm{~K})$ según sea el proceso ZFC o $\mathrm{FC}$, respectivamente.

Por otro lado, la susceptibilidad magnética de un grano esférico $100 \%$ superconductor debería alcanzar el valor expresado por $-1 / 4 \pi \mathbf{d}$ con unidades de emu/g donde $\mathbf{d}$ es la densidad de la fase superconductora obtenida por medio de la difracción de rayos $X$ [4]. Para nuestro compuesto la densidad determinada por los rayos $\mathrm{X}$ es igual a $6.518 \mathrm{~g} / \mathrm{cm}^{3}$ y el $100 \%$ del volumen superconductor corresponde a una magnitud de $-13.8 \times 10^{-3} \mathrm{emu} / \mathrm{g}-\mathrm{Oe}$ a la temperatura de $10 \mathrm{~K}$. Sin embargo el modelo utilizado requiere de partículas esféricas con un factor desmagnetizante expresado por: $D=4 \pi / 3$,

situación que no es real en nuestras muestras en polvo, pero aún la medida de la susceptibilidad magnética es una técnica importante para calcular el volumen superconductor o fracción Meissner. La comparación del valor experimental de la susceptibilidad magnética DC o AC, $\chi_{\text {exp }}$, con su valor teórico $-\frac{1}{4 \pi}$ es igual a:

$$
4 \pi \cdot \chi_{\exp }^{\prime}=-1.5
$$

Entonces con las magnitudes de la susceptibilidad magnética teórica (modelos de esferas) y experimental, utilizando la curva FC de la figura 3a y la ecuación 6 , obtenemos una fracción Meissner igual a $56.6 \%$ a la temperatura de $10 \mathrm{~K}$. Además, procediendo de manera semejante como en caso de la muestra de $\mathrm{Pb}$, el porcentaje del blindaje diamagnético obtenido de la curva ZFC de la figura 3 a es igual a $14 \%$ a $10 \mathrm{~K}$.
Un superconductor granulado bien preparado no contiene ninguna fase en estado normal, pero tiene espacios intergranulares y aún intragranulares, los cuales pueden anclar el flujo magnético. Un momento magnético determinado por un proceso de enfriamiento $\mathrm{FC}$ es significativamente menor que el momento ZFC, como es mostrado en la figura $3 \mathrm{a}$.

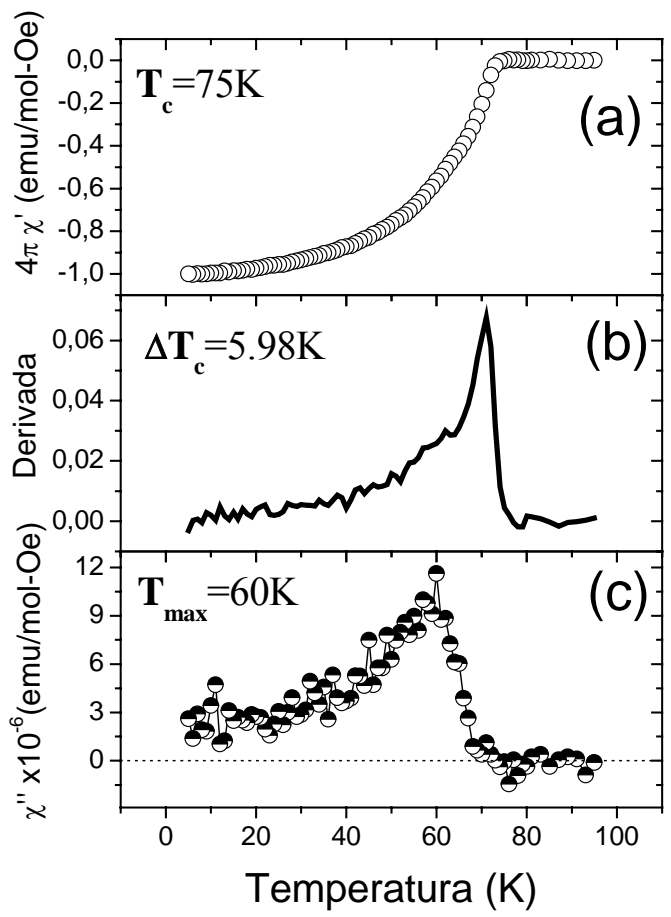

Fig. 4. Susceptibilidad magnética AC en un proceso ZFC para la muestra superconductora en polvo $\left[\mathrm{Y}_{0.75} \mathrm{Ca}_{0.25}\right](\mathrm{Ba}, \mathrm{Sr}) \mathrm{Cu}_{2.80}\left(\mathrm{PO}_{4}\right)_{0.20} \mathrm{O}_{\mathrm{z}}$ (a) parte real de la susceptibilidad AC, (b) derivada de curva (a), y (c) parte imaginaria de la susceptibilidad AC.

Las medidas de susceptibilidad magnética AC del óxido cerámico $\left[\mathrm{Y}_{0.75} \mathrm{Ca}_{0.25}\right](\mathrm{Ba}, \mathrm{Sr}) \mathrm{Cu}_{2.80}\left(\mathrm{PO}_{4}\right)_{0.20} \mathrm{O}_{\mathrm{z}}$ en polvo, se ilustran en la figura 4, con una intensidad de campo magnético externo aplicado de 2 Oe en un proceso de enfriamiento ZFC, el compuesto presenta un peso molecular de $597.79 \mathrm{~g} / \mathrm{mol} \mathrm{y}$ una masa analizada de $0.050 \mathrm{~g}$. El ancho finito de la transición al estado superconductor tiene una magnitud de $5.98 \mathrm{~K}$ como se ilustra en la figura 4b. Además el porcentaje del blindaje diamagnético obtenido de la figura $4 \mathrm{a}$ es igual a $23 \%$ a la temperatura de $5 \mathrm{~K}$. Se considera que las corrientes del blindaje diamagnético deben fluir no solo en la capa superficial sino también en las capas internas, dicho comportamiento es característico de muestras sintetizadas como es nuestro caso. 
El interés en estudiar esta muestra mediante la susceptibilidad magnética AC ha sido la comprensión de la relación existente entre la densidad de corriente crítica superconductora y la intensidad de campo magnético interno con la inflexión que sufre la señal $\chi^{\prime \prime}(T)$. Encontramos que el máximo en la curva $\chi^{\prime \prime}(T)$ (pico de pérdida de energía) empieza a la misma temperatura que la curva $4 \pi \cdot \chi^{\prime}(T)$, ésta se encuentra a una temperatura de $60 \mathrm{~K}$ muy cerca de la mitad (-0.56) del valor de la señal diamagnética en la curva $4 \pi \cdot \chi^{\prime}(T)$. El máximo de la curva está relacionado con la siguiente situación: cuando la densidad de corriente crítica superconductora y la intensidad de campo magnético interno alcanzan el centro de la muestra [9]. El campo magnético externo aplicado AC produce flujos magnéticos que alternativamente entran y salen de la muestra en la forma de vórtices En una muestra granular, como es nuestro caso, Gömöry [9] propone el siguiente modelo para explicar las inflexiones en la señal diamagnética: dentro de los granos superconductores la dinámica de los flujos está gobernada por la fuerza de anclaje intragranos caracterizada por la densidad de corriente crítica intragranos, mientras el sistema de contactos intragranos forman un efectivo medio de anclaje de los flujos, caracterizado por la densidad de corriente crítica intergranos.

Por otro lado, los tratamientos térmicos tienen una directa relación con la señal diamagnética como lo señala Miu [10] en muestras de $\mathrm{YBa}_{2} \mathrm{Cu}_{3} \mathrm{O}_{7}$ donde observa que tratamientos térmicos prolongados en oxígeno conlleva a un sustancial incremento de la señal diamagnética y una aguda transición al estado superconductor, debido al decrecimiento de las cantidades de defectos y al desarrollo de contactos del tipo Josephson entre las regiones superconductoras homogéneas en la muestra.

c) Muestra de YBCO en polvo y capa delgada: La figura 5 muestra los valores de la susceptibilidad magnética DC y el ancho de la transición al estado superconductor de una muestra en polvo de $\mathrm{YBa}_{2} \mathrm{Cu}_{3} \mathrm{O}_{7}$ Sol-Gel y una muestra de $\mathrm{YBa}_{2} \mathrm{Cu}_{3} \mathrm{O}_{7}$ en forma de película delgada. El interés de estudiar estas dos muestras de $\mathrm{YBa}_{2} \mathrm{Cu}_{3} \mathrm{O}_{7}$ ha sido comparar la forma de la señal diamagnética y el ancho de la transición al estado superconductor que está relacionado con los enlaces débiles y la anisotropía de los granos.

La masa utilizada en la muestra de $\mathrm{YBa}_{2} \mathrm{Cu}_{3} \mathrm{O}_{7}$ Sol-Gel fue de $0.062 \mathrm{~g}$, con una intensidad de campo magnético externo aplicado 20 Oe en un proceso ZFC, presentando un peso molecular $666.13 \mathrm{~g} / \mathrm{mol}$. Aunque el proceso de obtención de las dos muestras de $\mathrm{YBa}_{2} \mathrm{Cu}_{3} \mathrm{O}_{7}$ fue diferente, ambas presentan una temperatura crítica superconductora igual a $92 \mathrm{~K}$ como se aprecia en las figuras $5 \mathrm{a}$ y $5 \mathrm{c}$, pero los anchos de transición al estado superconductor fueron totalmente diferentes, $0.79 \mathrm{~K}$ y $5.6 \mathrm{~K}$, para las muestras en forma de capa delgada y polvo, respectivamente, como se observa en la figura $5 \mathrm{~b}$ y $5 \mathrm{~d}$. La diferencia en el ancho de la transición y la forma de la curva de la señal diamagnética está relacionada con la calidad de los granos superconductores (sin impurezas ni fases precursoras presentes), la textura de los granos superconductoras (textura en el plano y fuera del plano) pues las supercorrientes fluyen en direcciones paralelas a los planos superconductores de $\mathrm{CuO}_{2}$ (véase figura 1 de la referencia [5]).

El porcentaje del blindaje diamagnético obtenido para el $\mathrm{YBa}_{2} \mathrm{Cu}_{3} \mathrm{O}_{7}$ Sol-Gel es igual a $3.1 \%$ a la temperatura de $5 \mathrm{~K}$. Además, por la forma de la saturación de la señal diamagnética de las figuras $5 \mathrm{a}$ y $5 \mathrm{c}$, es claro que el flujo magnético es excluido muy marcadamente en la muestra en forma de película delgada. Este valor es muy diferente con respecto a las otras dos muestras debido a la magnitud de la intensidad de campo magnético externo aplicado que fue 10 veces mayor para la muestra de $\mathrm{YBa}_{2} \mathrm{Cu}_{3} \mathrm{O}_{7}$ en polvo.

La descripción de una muestra granular mediante la presencia de una red de enlaces débiles [11] o uniones del tipo Josephson se cree que es la responsable del comportamiento magnético en muestras sintetizadas de óxidos superconductores [12], además si consideramos la anisotropía del compuesto $\mathrm{YBa}_{2} \mathrm{Cu}_{3} \mathrm{O}_{7}$, uno obtiene magnitudes pequeñas de la densidad de corriente crítica superconductora en una muestra granular igual a $100 \mathrm{~A} / \mathrm{cm}^{2}$ con respecto al valor de la capa delgada $1 \mathrm{MA} / \mathrm{cm}^{2}$ a la temperatura de $77 \mathrm{~K}$.

Por tanto, para las aplicaciones tecnológicas de los superconductores de alta Tc es necesario el crecimiento en forma de película delgada 
controlando la textura biaxial de los granos superconductores [6].
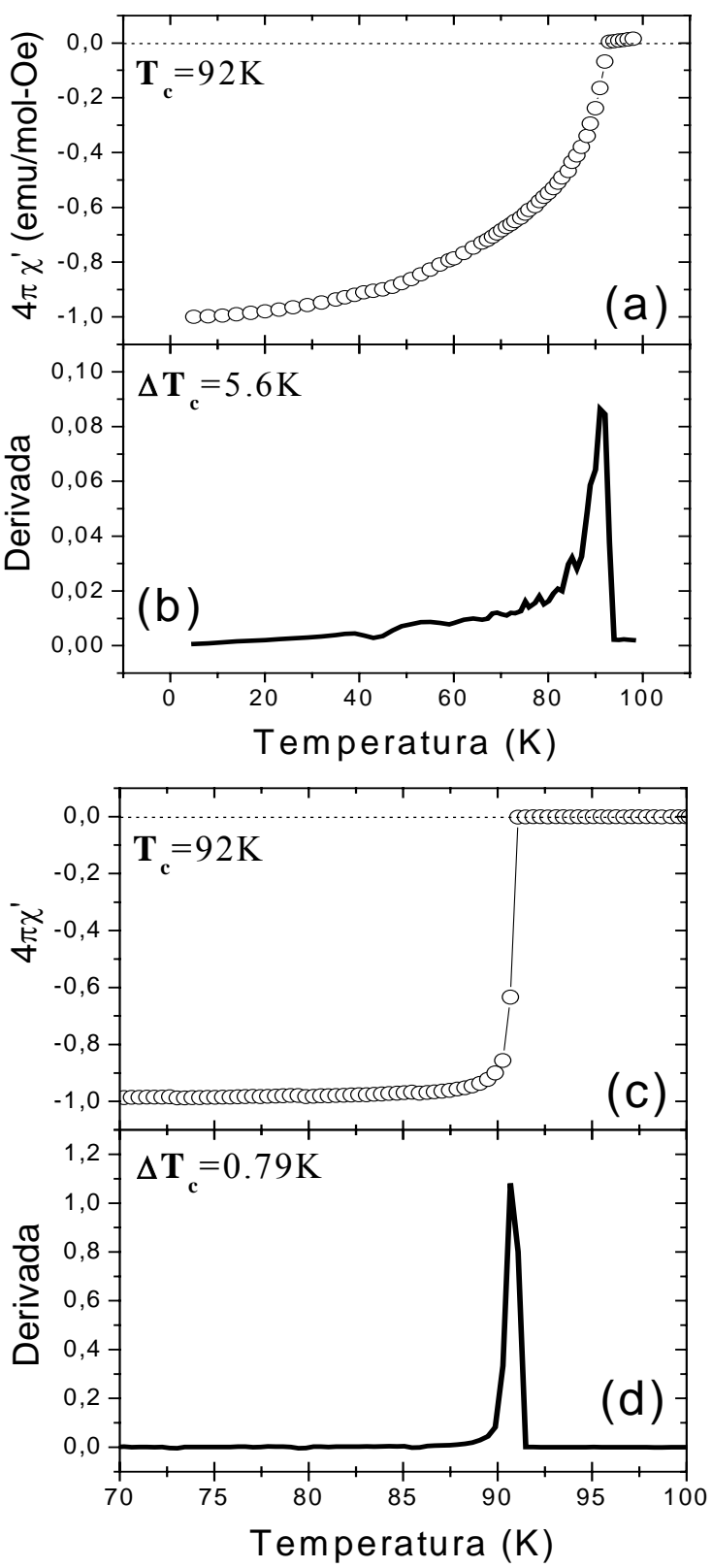

Fig. 5. (a) Susceptibilidad magnética DC de una muestra en polvo de $\mathrm{YBa}_{2} \mathrm{Cu}_{3} \mathrm{O}_{7}$ en un proceso $\mathrm{ZFC}$, (b) primera derivada de la curva de susceptibilidad magnética, (c) susceptibilidad magnética DC de una capa delgada de $\mathrm{YBa}_{2} \mathrm{Cu}_{3} \mathrm{O}_{7}$ con textura biaxial crecida sobre monocristal de $\mathrm{LaAlO}_{3}$; (d) primera derivada de la figura (c), obsérvese el agudo ancho de la transición $\sim 0.79 \mathrm{~K}$.

4.2 Estado Normal $(\mathrm{T}>\mathrm{Tc})$ :

a) Muestras en polvo conteniendo fosfatos: $\mathrm{La}$ figura 6a exhibe las medidas de la susceptibilidad DC en el estado normal de muestras de $\left[\mathrm{Y}_{0.75} \mathrm{Ca}_{0.25}\right](\mathrm{Ba}, \mathrm{Sr}) \mathrm{Cu}_{3-\mathrm{x}}\left(\mathrm{PO}_{4}\right)_{\mathrm{x}} \mathrm{O}_{\mathrm{z}}$ en polvo dopadas con grupos de fosfatos $(\mathrm{x}=0.0$, $0.15,0.30)$ con una intensidad de campo magnético externo aplicado igual a $20 \mathrm{kOe}$. El campo externo fue intenso para poder alinear todos los momentos magnéticos presentes en las muestras, señalamos que solamente hemos considerado los momentos magnéticos que se encuentran localizados en los sitios de $\mathrm{Cu}(2)$ [13] en los planos superconductores pues los átomos dopantes $\mathrm{Ca}$ y $\mathrm{P}$ no son magnéticos.

Las medidas de susceptibilidad en el estado normal fueron ajustadas utilizando la ecuación

$$
\chi=\chi_{o}+\frac{C}{\left(T-\theta_{c}\right)}
$$

donde $\chi_{0}$ es la susceptibilidad magnética independiente de la temperatura, $\mathrm{C}$ es la constante de Curie y $\theta_{c}$ es la temperatura de Curie-Weiss; como ejemplo se presenta un ajuste lineal en la figura 6a. Todas ellas siguieron un comportamiento descrito por la ley de CurieWeiss (ecuación 8). La constante de Curie disminuye con el incremento de grupos de fosfatos (figura 6b), hasta un 20\% con valores muy cercanos a cero. Por otro lado, la magnitud del término independiente de la temperatura $\chi_{o}$ no muestra una variación manteniéndose constante alrededor de $2 \times 10^{-4} \mathrm{emu} / \mathrm{mol}-\mathrm{Oe}$ lo que implica que no hay interacción magnética entre los momentos localizados en los sitios de $\mathrm{Cu}(2)$. La contribución de $\chi_{o}$ debida a los electrones de conducción en la superficie de Fermi. Los electrones que se encuentran en el dominio $\mathrm{k}_{\mathrm{B}} \mathrm{T}$ de la parte superior de la distribución de Fermi pueden orientarse con el campo magnético externo aplicado, además no contribuyen significativamente a la susceptibilidad magnética en el estado normal en las muestras bajo estudio.

El dopaje con el grupo de fosfatos no introduce momentos magnéticos localizados en la posición del $\mathrm{Cu}(1)$, como puede observarse en la figura $6 \mathrm{c}$ del valor del momento magnético efectivo es constante alrededor de 0.67. Dividiendo los valores de momento magnético efectivo entre las moles de cada uno de los compuestos, obtenemos de la constante $\mathrm{C}$ se encuentran en el rango $0.53-0.70 \mu_{\mathrm{B}} / \mathrm{mol}$ para el ión de $\mathrm{Cu}(2)$, los cuales son mayores por un factor 2 en comparación con el valor teórico de $0.3 \mu_{\mathrm{B}} / \mathrm{mol}$ para el ión de $\mathrm{Cu}(2)$ del compuesto $\mathrm{YBa}_{2} \mathrm{Cu}_{3} \mathrm{O}_{7}$. $\mathrm{La}$ diferencia radica en el contenido total de oxígeno en la muestra por la incorporación de grupo $\left(\mathrm{PO}_{4}\right)^{3-}$ además de generar números de 
coordinación 4 y 5 para el átomo de $\mathrm{Cu}(2)$ [4] en los planos superconductores de $\mathrm{CuO}_{2}$.

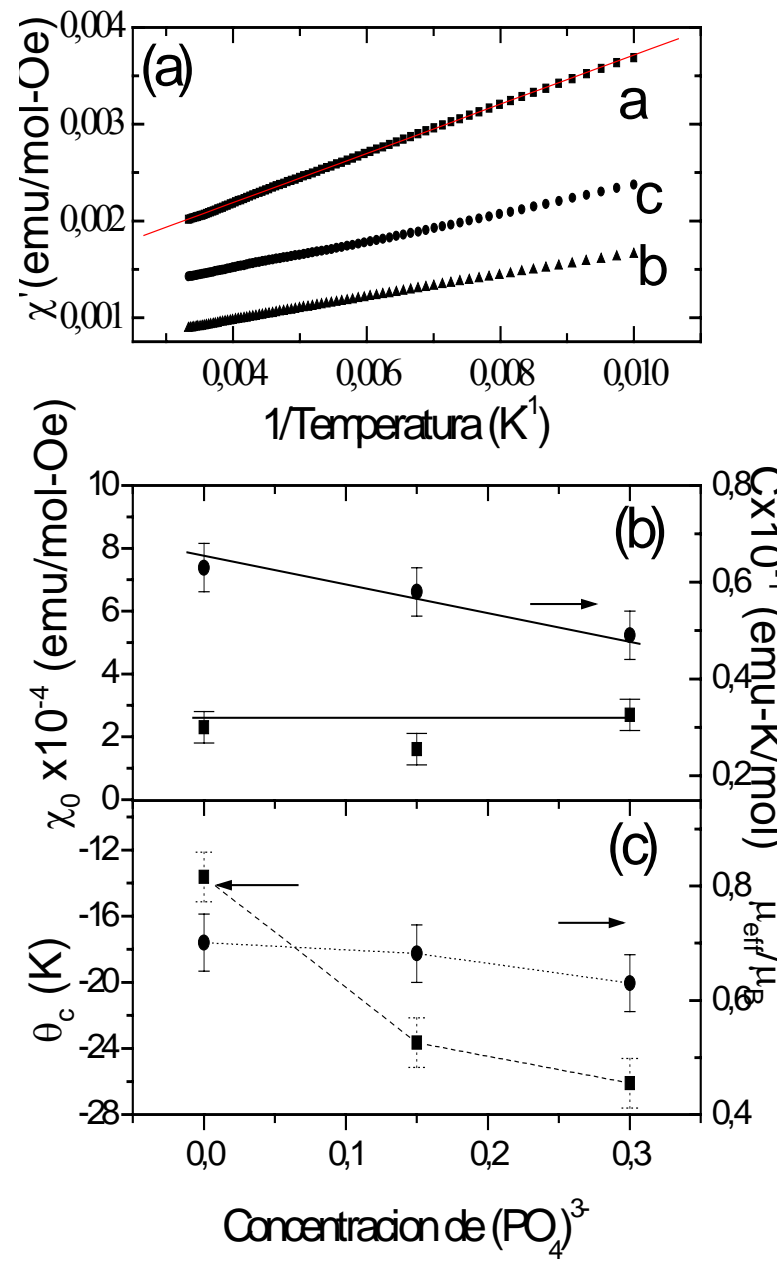

Fig. 6. Susceptibilidad en el estado normal de una muestra superconductora en polvo $\left[\mathrm{Y}_{0.75} \mathrm{Ca}_{0.25}\right](\mathrm{Ba}, \mathrm{Sr}) \mathrm{Cu}_{3-\mathrm{x}}\left(\mathrm{PO}_{4}\right)_{\mathrm{x}} \mathrm{O}_{\mathrm{z}}$ para tres dopajes $\mathrm{a}=0.0, \quad \mathrm{~b}=0.15$ y $\mathrm{c}=0.30$. (a) Comportamiento lineal de $\chi^{\prime}$ en función de $1 / \mathrm{T}$, (b) y (c)

parámetros $\chi_{o}, \mathrm{C}, \theta_{C} \mathrm{y} \mu_{\mathrm{eff}}$ determinados del ajuste lineal de $\chi^{\prime}$ en (a).

Finalmente los valores negativos de la temperatura de Curie-Weiss indican un acople paramagnético de los átomos de cobre $\mathrm{Cu}(2)$ de los planos superconductores. El incremento de la temperatura $\theta_{C}$ hacia valores más negativos se encuentra correlacionado con el contenido de oxígeno en las muestras, es decir, la remoción de átomos de oxígeno en las cadenas metálicas de $\mathrm{CuO}$ los cuales afectan la química de los bloques de reserva de carga que se encuentran directamente correlacionados con los valores de la $\mathrm{Tc}$ en el estado superconductor.

\section{Conclusiones}

La susceptibilidad magnética AC permite el estudio de la dinámica de un sistema magnético, si bien solapa la información que obtenemos de una medida DC, su importancia recae en la información adicional que uno puede conseguir de las propiedades del material bajo análisis.

La parte real de la susceptibilidad magnética AC representa el flujo excluido conjuntamente por las regiones intragranular e intergranular, mientras que la parte imaginaria de la susceptibilidad magnética $\mathrm{AC}$, es el resultado de la absorción del flujo dentro de las regiones de enlaces débiles formadas por el espacio intergranular, la curva muestra un máximo que representa la absorción del flujo.

La naturaleza granular de los superconductores en polvo estudiados tienen una saturación de la señal diamagnética solamente a bajas temperaturas $(<10 \mathrm{~K})$, debido a la presencia de una red de enlaces débiles o uniones del tipo Josephson que son responsables del comportamiento magnético.

La característica peculiar de los sistemas de alta Tc estudiados es que son sistemas granulares. En general contienen dos regiones a temperaturas por debajo de la Tc, la región de los granos superconductores y la región de las fronteras de grano que sirven como enlaces débiles.

La magnitud de la densidad de corriente crítica superconductora es menor en las muestras granulares debido a su orientación al azar de los granos superconductores, por lo cual, es necesario un ordenamiento de los mismos para lograr una textura biaxial y tener magnitudes de $\mathrm{J}_{\mathrm{c}}>1 \mathrm{MA} / \mathrm{cm}^{2}$ para aplicaciones tecnológicas.

Las muestras granulares en la región normal muestran un comportamiento de la susceptibilidad que sigue un comportamiento de la ley Curie-Weiss. Presentando un acople paramagnético de los átomo de $\mathrm{Cu}(2)$ en los planos superconductores de $\mathrm{CuO}_{2}$.

\section{Agradecimientos}

Los autores agradecen al Consejo Superior de Investigaciones de la UNMSM por el apoyo económico brindado a los proyectos de 
investigación de código 050702021, 060701021 y 071301021. También agradecemos al Prof. Xavier Obradors del ICMAB - CSIC (España) por permitirnos el uso del magnetómetro del tipo MPMS para las medidas DC. Así mismo, al Prof. C. W. Chu del Texas Center for Superconductivity de la Universidad de Houston (EEUU) por permitirnos el uso del magnetómetro del tipo PPMS para las medidas AC.

\section{Referencias}

[1] McEntee J. Chemistry World. 5 (2008) 42 - 47.

[2] Hott R. "Materials Aspects of High- Temperature Superconductors for Applications". Página 1 en High Temperature Superconductivity 1. A. V. Narlikar Editor. Springer Verlag. 2004.

[3] J. Crangle. "Solid State Magnetism". Capítulo 4, página 57. Edward Arnold Ed. 1991.

[4] J. C. González. Tesis de Maestría. "Preparación y Caracterización de Cerámicas Superconductoras del Sistema 123 conteniendo Fosfatos". Facultad de Ciencias Físicas. Unidad de Post Grado. Universidad Nacional Mayor de San Marcos. Agosto 2002. Lima - Perú.

[5] A. Bustamante, A. Osorio, J. C. González, M. Carhuancho, N. Salas, L. De Los Santos, N. De La Cruz. y A. Díaz. Rev. Per. Quím. Ing. Quím. 7 (2004) 3-8.
[6] J. C. González. Tesis de Doctorado. "Coated Conductors and Chemical Solution Growth of YBCO Films: A Micro-Raman Spectroscopy Study". Institut de Ciència de Materials de Barcelona - CSIC. Universitat Autònoma de Barcelona. Febrero 2005. Barcelona - España.

[7] A. H. Morrish. "The Physical Principles of Magnetism”. Capítulo 1, página 1. John Wiley \& Sons Ed. 1965.

[8] C. P. Poole Jr., H. A. Farach and R. J. Crewick. "Superconductivity". Capítulo 2, página 21. Ed. Academic Press. 1995.

[9] F. Gömöry. Supercond. Sci. Technol. 10 (1997) $523-542$.

[10] L Miu., S. Popa, M. Popescu and E. Z. Pavel. Phys. B. 70 (1988) $421-424$.

[11] K. Sato, T. Hikata, M. Mukai, M. Ueyama, N. Shibuta, T. Kato, T. Masuda, M. Nagata, K. Iwata and T. Mitsui. IEEE Trans. Magn. MAG27 (1991) 1231.

[12] S. Gotoh. "Magnetic properties II AC Magnetization" Capítulo 7, página 133 en "Melt Processed High- temperature Superconductors". Edited by M. Murakami. World Scientifc Publishing Co. 1992.

[13] N. M. Plakida. "High-Temperature Superconductivity: Experiment and Theory". Capítulo 3, página 43. Ed. Springer - Verlag. 1995. 3 